\title{
Antioxidant properties of breads prepared with $\beta$-glucan and black tea
}

\author{
A.M.M. Jalil, E. Combet, C.A. Edwards and A.L. Garcia \\ Human Nutrition, School of Medicine, College of Medical, Veterinary \& Life Sciences University of Glasgow, \\ New Lister Building, Glasgow Royal Infirmary, 10-16 Alexandra Parade, Glasgow, G31 2ER, Scotland, \\ United Kingdom
}

Tea is one of the most common beverages consumed worldwide. Short-term intake of teas reduced postprandial glycaemia in humans ${ }^{(1)}$. These effects have been attributed to the presence of bioactive polyphenols ${ }^{(2)}$ and have been linked to antioxidant properties. $\beta$-glucan is a soluble dietary fibre with mixed $\beta-(1 \rightarrow 3)$ and $\beta$ - $(1 \rightarrow 4)$ linkages. The effects of $\beta$-glucan on reducing post-prandial glycaemic and insulinaemic responses in humans are well established ${ }^{(3)}$. The synergistic effects of both bioactive components as part of a meal such as bread are promising. Food constituents can interact in several ways when eaten separately but if cooked together in a product the food matrix interactions may be more complex. The addition of polyphenols and/or dietary fibres to foods may directly or indirectly modify their digestibility and antioxidant properties ${ }^{(4,5)}$. Thus, elucidating mechanisms for such interactions is important before conducting in vivo studies. We hypothesized that the food matrix interactions of soluble fibre and tea polyphenols in bread will result in higher antioxidant activity in in vitro. Breads (white bread, WB; black tea, BT; $\beta$-glucan, BG; $\beta$-glucan plus black tea, BGT) were prepared using standard bread making techniques. Breads containing $0.5 \mathrm{~g}$ of available carbohydrate (dry weight) and wheat flour (WF) (control) were subjected to in vitro digestion (gastric and small intestinal models). An aliquot was taken after adding amylase $(0 \mathrm{~h})$, pepsin $(2 \mathrm{~h})$, pancreatic enzymes and bile extract $(5 \mathrm{~h})$, and after removal small molecular weight $(<1000 \mathrm{kDa})$ digestive products were used for the determination of antioxidant potential (FRAP) and total phenolic content (Folin-Ciocalteau method).
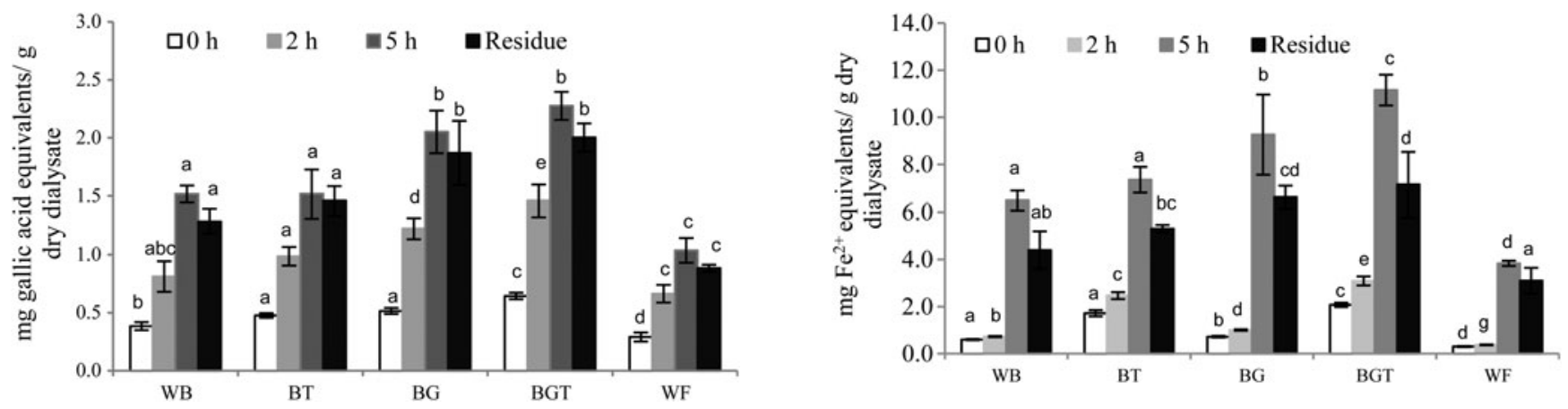

Values are mean (error bar represents the SD) for three replicates $(n$ 3). Different superscripts indicate significant values within the same time point (ANOVA followed by LSD post hoc test).

Total phenolic content of digested breads are shown in Fig. 1. The addition of amylase $(0 \mathrm{~h})$ significantly increased phenolic content of BGT compared with the other breads. Pepsin digestion $(2 \mathrm{~h})$ further increased phenolic content of BG and BGT compared with $\mathrm{WB}, \mathrm{BT}$ and SF. There was no significant difference in phenolic content of BG and BGT after pancreatin digestion (5 h) and after dialysis $(6 \mathrm{~h})$ compared with the other breads. BT and BGT showed significantly higher $(p<0 \cdot 05)$ FRAP activity after pepsin digestion with 2.5 to $3.1 \mathrm{mg} \mathrm{Fe}^{2+}$ equivalents/g dry dialysate compared with WB and BT $\left(0.7\right.$ to $1.0 \mathrm{mg} \mathrm{Fe}^{2+}$ equivalents/g dry dialysate $)$ (Fig. 2). Digestion with pancreatin significantly increased $(p<0.05)$ FRAP activity of BG and BGT compared to WB and BT. FRAP activity of BGT was similar to BG after dialysis $(6 \mathrm{~h})$ but remained higher than WB and BT. The results suggests that in vitro digestion with amylase, pepsin and pancreatin released substantial amount of phenolic from breads which could potentially being absorb in the small intestine for secondary metabolism but this needs further investigation.

1. Bryans JA, Judd PA, Ellis PR (2007) J Am Coll Nutr 26, 471-477.

2. Rothwell JA, Urpi-Sarda M, Boto-Ordonez M, et al. (2012) Database 2012, bas031-bas.

3. Ostman EM, Frid AH, Groop LC, et al. (2006) Eur J Clin Nutr 60, 334-341.

4. Juntunen KS, Laaksonen DE, Autio K, et al. (2003) Am J Clin Nutr 78, 957-964.

5. Rosen LA, Ostman EM, Bjorck IM (2011) J Agric Food Chem 59, 12149-12154. 